\title{
Phosphorylation of Synapsin I by cAMP-Dependent Protein Kinase Controls Synaptic Vesicle Dynamics in Developing Neurons
}

\author{
Dario Bonanomi, ${ }^{1}$ Andrea Menegon, ${ }^{1}$ Annarita Miccio, ${ }^{2}$ Giuliana Ferrari, ${ }^{2}$ Anna Corradi, ${ }^{3}$ Hung-Teh Kao, ${ }^{4}$ \\ Fabio Benfenati, ${ }^{3}$ and Flavia Valtorta ${ }^{1}$ \\ ${ }^{1}$ Department of Neuroscience, San Raffaele Scientific Institute and Vita-Salute University, 20132 Milan, Italy, ${ }^{2}$ Telethon Institute for Gene Therapy, San \\ Raffaele Scientific Institute, 20132 Milan, Italy, ${ }^{3}$ Department of Experimental Medicine, Section of Physiology, University of Genova, 16132 Genova, Italy, \\ and ${ }^{4}$ Nathan Kline Institute for Psychiatric Research, Orangeburg, New York 10962
}

In developing neurons, synaptic vesicles (SVs) undergo cycles of exo-endocytosis along isolated axons. However, it is currently unknown whether SV exocytosis is regulated before synaptogenesis. Here, we show that cAMP-dependent pathways affect SV distribution and recycling in the axonal growth cone and that these effects are mediated by the SV-associated phosphoprotein synapsin I. The presence of synapsin I on SVs is necessary for the correct localization of the vesicles in the central portion of the growth cone. Phosphorylation of synapsin I by cAMP-dependent protein kinase (protein kinase A) causes the dissociation of the protein from the SV membrane, allowing diffusion of the vesicles to the periphery of the growth cone and enhancing their rate of recycling. These results provide new clues as to the bases of the well known activity of synapsin I in synapse maturation and indicate that molecular mechanisms similar to those operating at mature nerve terminals are active in developing neurons to regulate the SV life cycle before synaptogenesis.

Key words: growth cone; phosphorylation; synapse formation; trafficking; knock-out mice; lentiviruses

\section{Introduction}

Synaptic release of neurotransmitter underlies neuronal communication in the adult brain. At the synapse, neurotransmitters stored in synaptic vesicles (SVs) are released during exocytosis, a process subjected to tight regulation through a complex array of protein-protein interactions and posttranslational modifications, most remarkably protein phosphorylation (Sudhof, 2004).

SV proteins are expressed before the establishment of synaptic contacts on vesicles that recycle constitutively along the axon and are eventually targeted to the nascent synapse, where they participate in the formation of a pool of recycling SVs (Matteoli et al., 1992; Ahmari et al., 2000). Over the past two decades, both spontaneous and evoked neurotransmitter release from growth cones of developing axons have been reported in a number of preparations (Young and Poo, 1983; Zakharenko et al., 1999). Thus, growth cones do not merely translate environmental cues into changes in membrane and cytoskeleton organization leading to axonal outgrowth and navigation but can also actively deliver messages to the external environment and integrate their responses with those of neighboring neurons. However, the mechanisms, if any, that regulate neurotransmitter secretion from developing axons remain to be explored.

Received Jan. 18, 2005; revised June 24, 2005; accepted June 25, 2005.

This work was supported by grants from the Italian Ministry of University (Cofin 2003 and 2004, University Excellence (enter on Physiopathology of (ell Differentiation, and Fondo Integrativo Ricerea di Base) and from Consiglio Nazionale delle Ricerche (Progetto Genomica Funzionale).

Correspondence should be addressed to Flavia Valtorta, DIBIT 3A3, San Raffaele Scientific Institute, via Olgettina 58, 20132 Milan, Italy. E-mail: valtorta.flavia@hsr.it.

DOI:10.1523/JNEUROSCI.1573-05.2005

Copyright $\odot 2005$ Society for Neuroscience $\quad$ 0270-6474/05/257299-10\$15.00/0
It has been shown previously that spontaneous neurotransmitter release from growth cones is regulated by protein kinase $\mathrm{A}$ (PKA), which also modulates neurotransmission at mature terminals (Trudeau et al., 1996; Yao et al., 2000). A striking qualitative similarity was reported between the PKA substrates of growth cone preparations from fetal rat brain and nerve terminal preparations from adult brain, the most notable example being the SV-associated protein synapsin I (SynI) (Ellis et al., 1985).

Synapsins are brain phosphoproteins that were discovered because of their prominent phosphorylation by PKA. Three synapsin genes have been identified and named synapsin I, II, and III. Transcripts of each of these genes are spliced differentially to give rise to various isoforms (for review, see Hilfiker et al., 1999). A single phosphorylation site (site 1) for PKA and $\mathrm{Ca}^{2+}$ / calmodulin-dependent kinase (CaMK) I/IV present at the $\mathrm{N}$-terminus is highly conserved among the various isoforms and across vertebrate and invertebrate species (Kao et al., 1999). Synapsin I is also phosphorylated by CaMK II (Huttner and Greengard, 1979), mitogen-activated protein kinase Erk (extracellular signal-regulated kinase), and cyclin-dependent kinase (Jovanovic et al., 1996). Synapsins have been proposed to maintain a reserve pool of vesicles in the proximity of the active zone by tethering SVs to each other and to the actin cytoskeleton, to control the availability of SV s through their ability to dissociate from SVs and actin in a phosphorylation-dependent manner, and to play a role in the postdocking steps of exocytosis (Valtorta et al., 1992; Benfenati et al., 1993; Hilfiker et al., 1998).

The axonal growth cone can be envisioned as a precursor of the presynaptic terminal and is therefore a privileged site to study the dynamics of SVs before synaptogenesis. In this study, we have 
addressed the mechanisms controlling the SV life cycle in growth cones associated with growing axons of embryonic hippocampal neurons in culture.

\section{Materials and Methods}

Materials. The polyclonal antibody against synaptophysin I (SypI) has been described previously (Valtorta et al., 1988). The anti-syntaxin 13 polyclonal antibody and anti-synaptotagmin I [cytoplasmic domain $\left(S_{\mathrm{C}}\right)$ and lumenal domain $\left(\mathrm{Syt}_{\mathrm{L}}\right)$ ] monoclonal antibody were from Synaptic Systems (Göttingen, Germany). The anti-synapsin I/II monoclonal antibody (clone 19.11), the anti-synapsin I polyclonal antibody G177, and the phosphorylation state-specific anti-synapsin polyclonal antibodies G257 (phosphosite-1) and RU19 (phosphosite-3) were prepared and characterized at The Rockefeller University (New York, NY) (Valtorta et al., 1988; Menegon et al., 2000). The phospho-synapsin (Ser9) antibody (phosphosite-1 specific) used in Western blotting was from Cell Signaling Technology (Beverly, MA). The anti-ERK antibody (K-23) was from Santa Cruz Biotechnology (Santa Cruz, CA). Tetramethylrhodamine isothiocyanate (TRITC)-, fluorescein isothiocyanate (FITC)-, and 7-amino-4-methylcoumarin-3-acetic acid-conjugated secondary antibodies and pure rabbit IgG antibodies were from Jackson ImmunoResearch (West Grove, PA). TRITC-conjugated phalloidin was from Sigma-Aldrich (Steinheim, Germany). Peroxidase-conjugated anti-rabbit was from Bio-Rad (Hercules, CA). The enhanced chemiluminescence detection system was from Amersham Biosciences (Little Chalfont, Buckinghamshire, UK), and BCA protein assay reagent was from Pierce (Rockford, IL). Cytochalasin D, H89, and forskolin were from Calbiochem (La Jolla, CA). $N^{6}, 2^{\prime}$-O-dibutyryladenosine $3^{\prime}, 5^{\prime}$ cyclic monophosphate (BT-cAMP) was from Sigma-Aldrich. FM 4-64 was from Molecular Probes (Eugene, OR). All chemicals were diluted in Krebs'-Ringer's-HEPES (KRH) [containing the following (in mM): 150 $\mathrm{NaCl}, 5 \mathrm{KCl}, 1.2 \mathrm{MgSO}_{4}, 1.2 \mathrm{KH}_{2} \mathrm{PO}_{4}, 2 \mathrm{CaCl}_{2}, 10$ glucose, and 10 HEPES/Na, pH 7.4] at the indicated concentrations.

Plasmid and virus production. The fragment SypI-enhanced yellow fluorescent protein (SypI-EYFP) was amplified by PCR from the plasmid pSypI-L-EYFP (Pennuto et al., 2002) using the primers 5'-GGGGACCGGTCAGCAGCAATGGACGTGGTG-3' and 5' GGGGGTCGACTTACTTGTACAGCTCGTCCATG-3'. After AgeISalI digestion, the SypI-EYFP insert was cloned into the selfinactivating lentiviral vector RRL.ppt.hPGK.GFP.pre.sin-18 (Lotti et al., 2002), in which enhanced green fluorescent protein (EGFP) was removed by an AgeI-SalI cut to produce pRRL-SypI-EYFP(pgk). The RRL.ppt.hPGK.GFP.pre.sin-18 plasmid contains a central polypurine tract (ppt) to enhance transduction in nondiving cells, the woodchuck hepatitis virus posttranscriptional regulatory element to increase expression of the targeted transgene, and a deletion in the U3 region of the long terminal repeat. Inserts are placed under the control of an internal, constitutive phosphoglycerol kinase (PGK) promoter. The fragment enhanced cyan fluorescent protein-vesicleassociated membrane protein 2 (ECFP-VAMP2) was extracted from pECFP-VAMP2 (Pennuto et al., 2002) by a NheI-NotI cut and cloned into pBS SK II (Stratagene, La Jolla, CA) digested with EcoRV after filling of the NheI and NotI sites to generate pBS SK ECFP-VAMP2. ECFP-VAMP2 extracted from pBS SK ECFP-VAMP2 by a XmaI-SalI cut was ligated into RRL.ppt.hPGK.GFP.pre.sin-18 digested with AgeI-SalI to generate pRRL-ECFP-VAMP2(pgk). The cDNAs of rat synapsin Ia and the S9A mutant were cloned into the plasmid pEGFP-C1 (Clontech, Palo Alto, CA). EGFP was substituted with ECFP extracted from ECFP-C1 by a NheI-BsrGI cut and ligation to produce the plasmids pECFP-Syn Ia and ECFP-Syn Ia S9A. ECFPsynapsin Ia and ECFP-synapsin Ia S9A were extracted from the respective plasmids by an AgeI-SalI digestion and cloned into the RRL.ppt.hPGK.GFP.pre.sin-18 plasmid to generate pRRL-ECFPSyn Ia(pgk) and pRRL-ECFP-Syn Ia S9A(pgk). The two digested fragments were also introduced into the plasmid pRRLsinPTT.CMV.GFPpre (Lotti et al., 2002), in which EGFP was removed by an AgeI-SalI cut to produce the pRRL-ECFP-Syn Ia(CMV) and pRRLECFP-Syn Ia S9A(CMV) plasmids, in which the inserts are placed under the control of the cytomegalovirus (CMV) promoter.
Viral stocks pseudotyped with the vesicular stomatitis G-protein (VSV-G) were prepared by transient cotransfection of $293 \mathrm{~T}$ cells with the transfer vector, the pCMV $\Delta$ R8.74 plasmid encoding Gag, Pol, Tat, and Rev, and the pMD.G plasmid encoding VSV-G, as described previously (Lotti et al., 2002). Viral preparations were concentrated by ultracentrifugation to increase titer. Viral titers (infectious particles) were determined by transduction of 293T cells with serial dilution of the viral stocks and evaluation of transduction efficiency by flow cytometry scoring of fluorescent cells.

Cell culture and transduction of neurons. Primary neuronal cultures were prepared from the hippocampi of embryonic day 18 embryos from Sprague Dawley rats (Charles River Italica, Calco, Italy), synapsin I knock-out mice (Li et al., 1995), or synapsin I/II/III triple knock-out mice (Feng et al., 2002) as described previously (Banker and Cowan, 1977). Four hours after plating of neurons, coverslips were placed in a clean dish containing glia-conditioned medium [MEM supplemented with 1\% N2 supplement (Invitrogen, San Diego, CA), 2 mm glutamine (BioWhittaker, Walkersville, MD), 0.1\% ovalbumin, $1 \mathrm{~mm}$ sodium pyruvate (Sigma-Aldrich), and $4 \mathrm{~mm}$ glucose] and incubated for $10-15 \mathrm{~h}$ at $37^{\circ} \mathrm{C}$ in a $5 \% \mathrm{CO}_{2}$ humidified atmosphere in the presence of viral supernatant at 1-10 multiplicity of infection. After transduction, neurons were returned to the original dishes and maintained in culture in glia-conditioned medium.

Immunoblot analysis. Neurons were rinsed rapidly with KRH devoid of $\mathrm{Ca}^{2+}$ and supplemented with 2 mM EGTA (KRH/EGTA), solubilized by scraping with solubilization buffer (1\% SDS, 2 mM EGTA, and $10 \mathrm{~mm}$ HEPES/Na, pH 7.4), and subjected to SDS-PAGE and immunoblotting as described previously (Menegon et al., 2002).

Cell labeling protocols. For standard double immunofluorescence analysis, cells were rinsed rapidly with KRH/EGTA, fixed for 30 min with $4 \%$ paraformaldehyde, $4 \%$ sucrose in $120 \mathrm{~mm}$ sodium phosphate buffer, $\mathrm{pH}$ 7.4, supplemented with 2 mM EGTA, and processed as described previously (Pennuto et al., 2003). TRITC-conjugated phalloidin was added during incubation of the secondary antibodies when indicated. For detection of synapsin phosphorylation, the cyanine 3-conjugated phosphosite-3-specific anti-synapsin I antibody (RU19) (Menegon et al., 2002) was added after the standard double-labeling protocol. Incubation with the conjugated antibody was performed in the presence of $10 \mu \mathrm{g} / \mathrm{ml}$ rabbit IgG and was preceded by a $20 \mathrm{~min}$ blocking reaction in the same solution. For the $\mathrm{Syt}_{\mathrm{L}}$-based exo-endocytic assay, neurons were incubated for 15 min with the Syt ${ }_{\mathrm{L}}$ antibody diluted in $\mathrm{KRH}$, washed rapidly with KRH/EGTA, fixed, detergent permeabilized, and counterstained with the second primary antibody followed by TRITC- and FITCconjugated secondary antibodies. For FM 4-64 uptake experiments, neurons were incubated with FM 4-64 $(10 \mu \mathrm{M})$ diluted in KRH for $1 \mathrm{~min}$ at room temperature (RT), washed three times by complete medium substitution with KRH during $1 \mathrm{~min}$, fixed, and processed for immunofluorescence.

Videomicroscopy and image analysis. Specimens were viewed with an Axiovert 135 inverted microscope (Zeiss, Oberkochen, Germany) equipped with epifluorescence optics. Images were recorded with a C4742-98 ORCA II cooled charge-coupled device camera (Hamamatsu Photonics, Hamamatsu City, Japan) and processed using Image Pro Plus 4.5 (Media Cybernetics, Silver Spring, MD) and Adobe Photoshop 6.0 (Adobe Systems, San Jose, CA).

For the evaluation of the colocalization index between SypI immunoreactivity and FM 4-64 signal, the percentage of SypI-positive pixels overlapping with FM 4-64-positive pixels was measured.

To quantify the degree of SV dispersion in growth cones, the area occupied by SVs (i.e., number of SV-positive pixels) was normalized to the total area of the growth cone as determined based on either filamentous actin (F-actin) staining or differential interference contrast (DIC) images. The threshold parameters adopted to automatically select SVpositive pixels using Adobe Photoshop 6.0 were chosen so to reveal continuity between SV-positive pixels over a wide range of signal intensities.

For the quantification of the $\mathrm{Syt}_{\mathrm{L}}$ antibody internalization, neurons double labeled with $S y t_{L}$ and $S y t_{C}$ recognized by TRITC- and FITCconjugated secondary antibodies, respectively, were imaged, and the intensities of the TRITC and FITC signals were quantified within the whole growth cone area by generating a binary mask based on the FITC signal 
$\left(\right.$ Syt $\left._{C}\right)$. The index of Syt $t_{L}$ internalization $\left[F\left(\right.\right.$ Syt $\left._{L}\right) / F\left(\right.$ Syt $\left.\left._{C}\right)\right]$ expresses the ratio between the TRITC and FITC intensities.

The pseudocolor spectrum surface plot showed in Figure 8 was generated using a dedicated command of Image Pro Plus 4.5 to obtain a threedimensional representation of the intensities of the SypI-EYFP fluorescence, with warmer hues corresponding to pixels of higher fluorescence intensity.

All experiments were repeated at least three times, and for each experimental point, the number of growth cones examined was in the range $15-100$.

\section{Results}

Spatial organization and dynamics of SVs in the axonal growth cone

To monitor the dynamics of SV protein-containing organelles in living neurons, we generated a fluorescent chimera of the SV marker SypI fused to EYFP and directed its expression in neurons through a lentiviral-mediated expression system (Lotti et al., 2002). To limit the possibility of altered sorting of the exogenous protein to organelles other than SVs, which is observed at high expression levels (Pennuto et al., 2003), the transgenes were placed under the control of a constitutive cellular promoter, the PGK promoter.

SypI-EYFP was associated with vesicles trafficked along the axon and concentrated in the central $(\mathrm{C})$ domain of the axonal growth cone, characterized by high organelle and microtubule density (Forscher et al., 1987). These vesicles appeared to be virtually absent from the peripheral $(\mathrm{P})$ domain of the growth cone, characterized by low organelle density and high F-actin content (Fig. $1 a, b$ ). Consistently, the vast majority of the vesicles bearing endogenous SypI ( $95 \pm 5 \%$; mean \pm SD; $n=20$ growth cones) were clustered in the core of the $\mathrm{C}$ domain but excluded from the $\mathrm{P}$ domain underlain by $\mathrm{F}$-actin. In agreement with previous studies, we will refer to organelles containing SV proteins before the formation of synaptic contacts as bona fide SVs (Matteoli et al., 1992; Sabo and McAllister, 2003).

To study membrane recycling in the growth cone, we used the styryl dye FM 4-64, which is internalized during endocytosis. After a brief ( $1 \mathrm{~min}$ ) incubation with the dye, intense fluorescent staining was associated with large membrane structures at the distal edge of the $\mathrm{C}$ domain. However, SypI-positive vesicles showed a limited overlap with the FM 4-64 signal (percentage of colocalization $\pm \mathrm{SD}, 9.5 \pm 4.1 ; n=15$ ), indicating a negligible rate of basal SV recycling, when compared with the high levels of constitutive endocytosis observed in the growth cone at this developmental stage (Fig. $1 c, c^{\prime}$ ).

To answer the question as to whether F-actin plays a role in the retention of SVs in the C domain, we exposed hippocampal neurons to cytochalasin D, which causes F-actin disassembly (Forscher and Smith, 1988; Contestabile et al., 2003). After such treatment, SVs marked by either SypI-EYFP or endogenous SypI were dispersed throughout the growth cone and reached the distal edge of the $\mathrm{P}$ domain (Fig. $1 d$ and data not shown). The percentage of growth cone area occupied by SypI-positive SVs before and after cytochalasin D treatment (mean \pm SD) was $19 \pm 5$ and $81 \pm$ $11 \%$, respectively $(n=16)$. Thus, disorganization of actin allows the movement of SVs into the P domain.

cAMP-modulated control of the distribution and recycling of SVs in the growth cone

Because cAMP was shown previously to affect organelle trafficking in the growth cone (Forscher et al., 1987; Hollenbeck, 1993), we wanted to determine whether cAMP-dependent pathways have a role in the control of the distribution of SVs in the $\mathrm{C}$
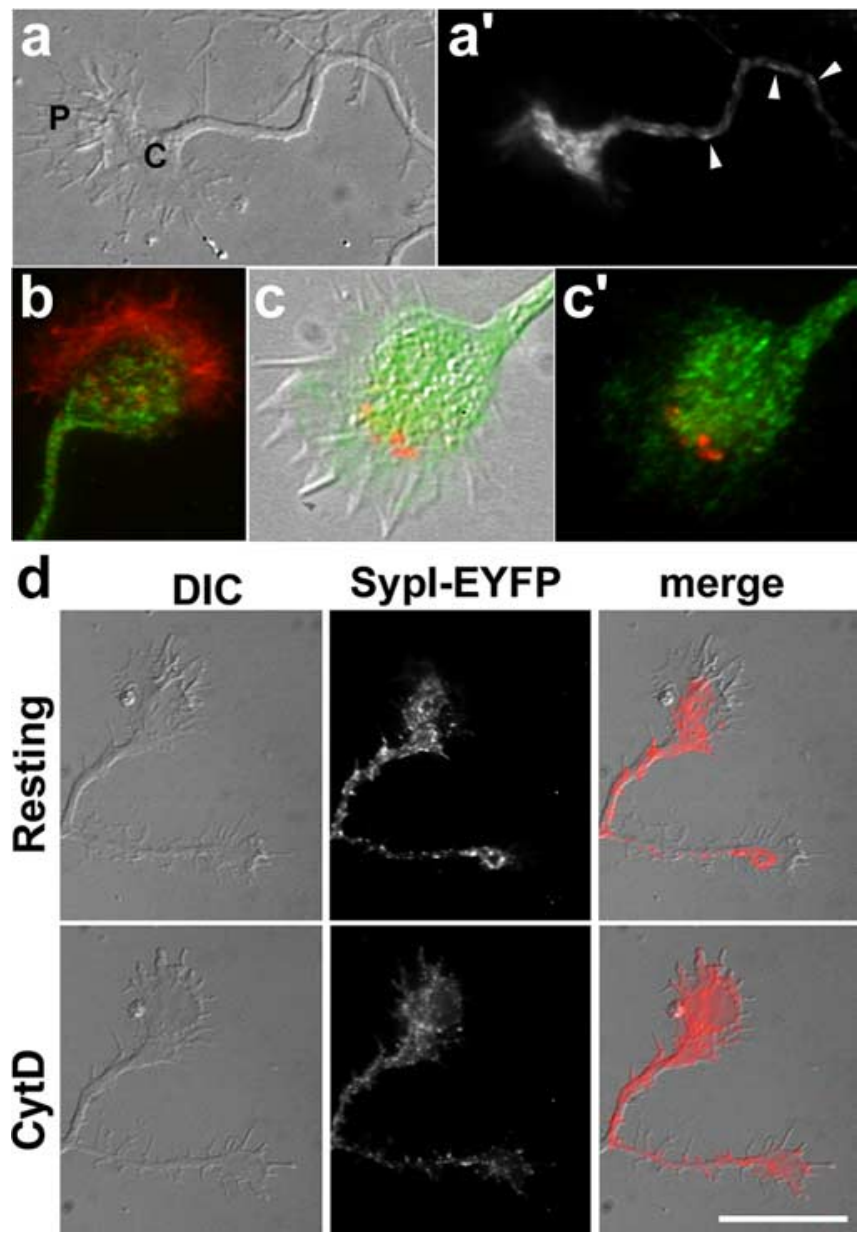

Figure 1. Localization of SVs in the core domain of the growth cone depends on the integrity of the F-actin meshwork. DIC $(\boldsymbol{a})$ and fluorescence $\left(\boldsymbol{a}^{\prime}\right)$ images of a living rat hippocampal neuron expressing Sypl-EYFP are shown. The chimera is associated with organelles distributed along the axon ( $\boldsymbol{a}^{\prime}$, arrowheads) and concentrated in the central domain of the growth cone $(C)$ but virtually absent in the peripheral domain (P). $\boldsymbol{b}$, Immunofluorescence of a growth cone labeled with an antibody against Sypl and TRITC-conjugated phalloidin to stain F-actin. Vesicles bearing endogenous Sypl (green) are clustered in the core of the growth cone and excluded from the $P$ domain enriched in F-actin (red). $c, c^{\prime}$, Basal uptake ofFM 4-64 (red) in a growth cone incubated with the dye for $1 \mathrm{~min}$, fixed, and retrospectively labeled with an anti-Sypl antibody (green). The overlay between the fluorescence $\left(\boldsymbol{c}^{\prime}\right)$ and DIC images is shown in c. FM 4-64 is internalized in large organelles at the interface between the $C$ and $P$ domains. $d$, Growth cones of rat hippocampal neurons expressing Sypl-EYFP were imaged before and after incubation with $10 \mu \mathrm{m}$ cytochalasin D (CytD) for 15 min at RT. In the right column, the overlay between the DIC and fluorescence (red) images is shown. At rest, Sypl-positive vesicles are concentrated in the $C$ domain, but they disperse throughout the growth cone after cytochalasin $D$ treatment. Scale bar: (in d) $\boldsymbol{a}, \boldsymbol{a}^{\prime}, 10 \mu \mathrm{m} ; \boldsymbol{b}, 8 \mu \mathrm{m} ; \boldsymbol{c}, \boldsymbol{c}^{\prime}, 6 \mu \mathrm{m}$; in $\boldsymbol{d}, 16 \mu \mathrm{m}$.

domain. Neurons expressing SypI-EYFP were imaged before and after incubation with forskolin, an adenylate cyclase activator that increases intracellular cAMP levels (Seamon et al., 1981). This treatment markedly altered SV distribution by inducing dispersion of SVs from the C domain throughout the growth cone. The changes in SV distribution occurred in the absence of major rearrangements in growth cone morphology, as shown by DIC images (Fig. 2, top). Treatment of neurons with the membranepermeant and phosphodiesterase-resistant cAMP analog BTcAMP (10 mM) for 7 min produced a comparable dispersion of SVs in growth cones (data not shown). In most of the growth cones analyzed, we also observed a reduction in the size of the fluorescent puncta representing SV clusters, suggesting a decrease in SV aggregation. 


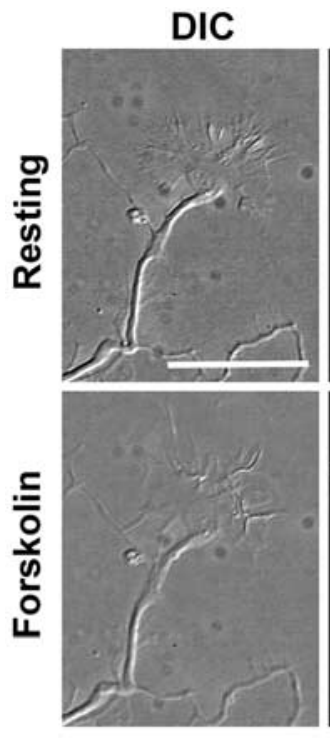

F-actin

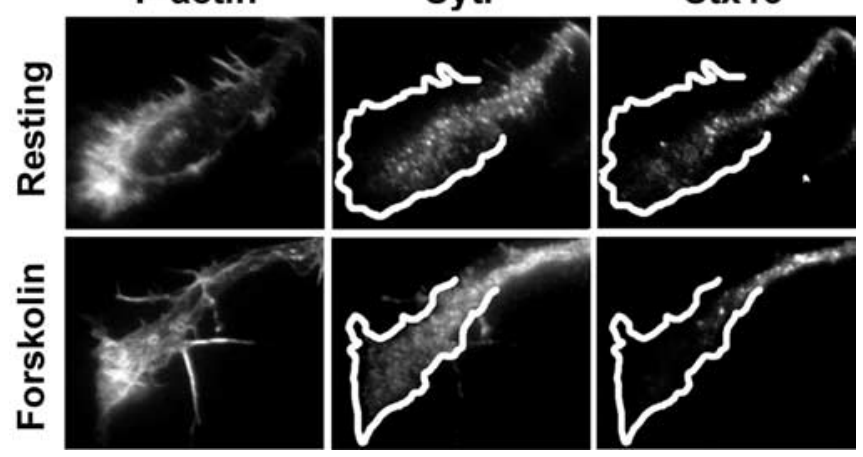

Figure 2. Elevation of intracellular cAMP leads to SV diffusion to the P domain. Top, Growth cone of a rat hippocampal neuron expressing Sypl-EYFP, imaged before and after incubation with $50 \mu \mathrm{m}$ forskolin for $10 \mathrm{~min}$ at RT. In the right column, the overlay between the DIC and fluorescence (red) images is shown. At rest, Sypl-positive vesicles are concentrated in the C domain, but they disperse throughout the growth cone after forskolin treatment. Bottom, Growth cones fixed either at rest or after treatment with forskolin for 5 min at $37^{\circ} \mathrm{C}$, stained with antibodies against synaptotagmin I (Sytl) and syntaxin 13 (Stx13) and with TRITC-conjugated phalloidin. The white trace outlines the distal edge of the P domain, as determined based on DIC images. Forskolin promotes the mobilization of synaptotagmin I-positive SVs to the $P$ domain, whereas syntaxin 13-positive vesicles are still retained in the C domain. No major change in the F-actin staining is visible after forskolin treatment. Scale bar: (in top panel) top, $12 \mu \mathrm{m}$; bottom, $8 \mu \mathrm{m}$.

The specificity of the effect of cAMP increase on the localization of SVs was tested by staining neurons treated with forskolin for the integral SV protein synaptotagmin I (Koh and Bellen, 2003), the endosomal antigen syntaxin 13 (Prekeris et al., 1999), and F-actin. Before forskolin treatment, both synaptotagmin I- and syntaxin 13-labeled vesicles were localized in the $\mathrm{C}$ domain and excluded from the $\mathrm{P}$ domain. The percentage of growth cone surface occupied by synaptotagmin I-positive SVs and syntaxin 13-labeled vesicles (mean \pm SD) was $21 \pm 2$ and $25 \pm 7 \%$, respectively $(n=15)$. Forskolin induced mobilization of synaptotagmin I-positive SVs into the P domain (average growth cone area occupancy $\pm \mathrm{SD}, 80 \pm 22 \% ; n=15)$, whereas syntaxin 13-positive endosomes remained confined to the $\mathrm{C}$ domain (average growth cone area occupancy $\pm \mathrm{SD}, 28 \pm 8 \%, n=15$ ) (Fig. 2 , bottom). F-actin staining was unaffected [mean phalloidin fluorescence $\pm \mathrm{SD}, 90 \pm 5.4$ and $101 \pm 2.6$ (arbitrary units) in resting and forskolin-treated growth cones, respectively; $n=30$ ].

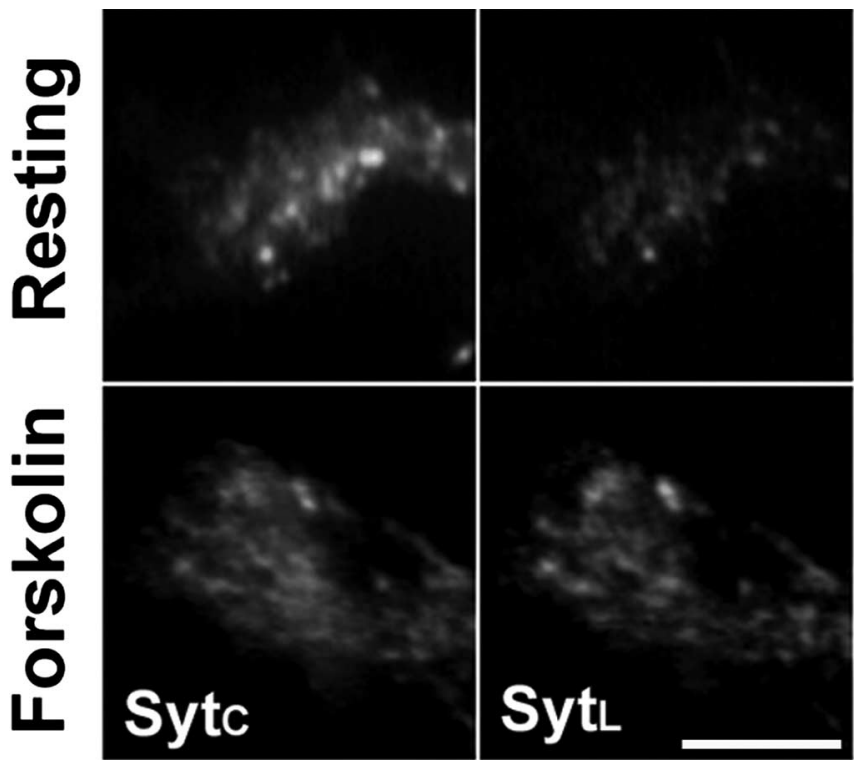

Figure 3. Elevation of intracellular cAMP enhances SV recycling in growth cones. Growth cones of neurons incubated at $37^{\circ} \mathrm{C}$ with an antibody directed against Syt for either $15 \mathrm{~min}$ in KRH or $5 \mathrm{~min}$ in $50 \mu \mathrm{m}$ forskolin followed by $10 \mathrm{~min}$ in KRH. After incubation, neurons were fixed and counterstained with an antibody against total synaptotagmin I (Syt ${ }_{c}$ ). Scale bar, $5 \mu \mathrm{m}$.

Because SVs diffused toward the growth cone membrane after forskolin treatment, we asked whether the rate of basal SV recycling was also modulated by intracellular cAMP levels. To accurately study exo-endocytosis of SVs occurring at low rates in the growth cone, we exploited an assay based on the uptake of an antibody directed against the lumenal domain of synaptotagmin I. This antibody binds to synaptotagmin I after exposure of the SV membrane at the cell surface and is then internalized by endocytosis of the SV membrane (Matteoli et al., 1992). Stage 3 neurons were incubated for 5 min with $S_{4} t_{L}$ in $\mathrm{KRH}$ in the presence or absence of forskolin, followed by an additional $10 \mathrm{~min}$ incubation with the antibody in $\mathrm{KRH}$. After treatment, neurons were counterstained with an antibody directed against the cytoplasmic domain of synaptotagmin I, and the levels of $S_{\mathrm{L}} \mathrm{L}_{\mathrm{L}}$ uptake were normalized to the total synaptotagmin I content (i.e., Syt ${ }_{\mathrm{C}}$ signal intensity) of each growth cone. The ratio between the fluorescence intensities of $S_{y} t_{L}$ and $S y t_{C}$ calculated for each growth cone was $0.35 \pm 0.11$ under control conditions and $0.50 \pm 0.12$ after treatment with forskolin (mean $\pm \mathrm{SD} ; n=43$; $p<0.001$; Student's $t$ test vs control growth cones). Thus, forskolin-induced SV mobilization was accompanied by a significant increase in the fraction of Syt $t_{L}$ internalized by the growth cones (Fig. 3), indicating that cAMP-dependent pathways control the basal recycling of SVs in growth cones of isolated axons.

\section{Synapsins associate with recycling SVs and are} phosphorylated by PKA in the growth cone

To identify and characterize the SV pool associated with synapsins in the growth cone, neurons were processed by double immunofluorescence for synaptotagmin I (to mark SVs) and total synapsin I. Bright fluorescent puncta of synapsin I immunoreactivity were associated with a fraction of the synaptotagmin I-positive SVs localized in the C domain, although low levels of diffuse synapsin I immunoreactivity were also present in both the $\mathrm{C}$ and $\mathrm{P}$ domains of the growth cone (Fig. $4 a{ }^{\prime \prime}$ ). A high degree of colocalization of synapsin I with synaptotagmin I was also de- 

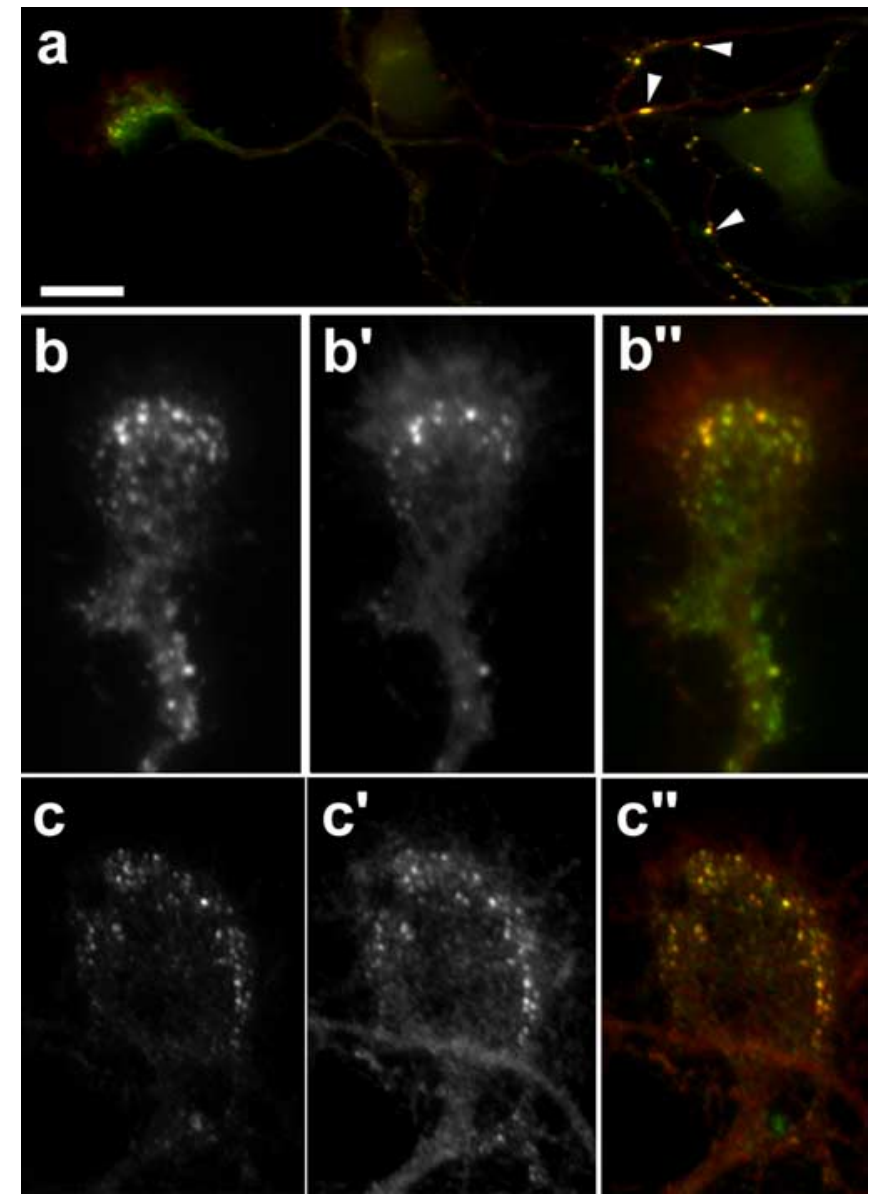

Figure 4. Synapsin associates with recycling SVs in growth cones. $\boldsymbol{a}-\boldsymbol{b}^{\prime \prime}$, Double immunofluorescence of rat hippocampal neurons stained with antibodies against synaptotagmin I ( $\boldsymbol{b}$ green in $\boldsymbol{a}$ and $\left.\boldsymbol{b}^{\prime \prime}\right)$ and synapsin I ( $\boldsymbol{b}^{\prime}$; red in $\boldsymbol{a}$ and $\left.\boldsymbol{b}^{\prime \prime}\right)$. Synapsin I colocalizes with synaptotagmin I in a subpopulation of SVs in the growth cone and at virtually all synaptic varicosities along the axon ( $\boldsymbol{a}$, arrowheads). $\boldsymbol{c}-\boldsymbol{c}^{\prime \prime}$, Neurons were incubated with the Syt antibody ( $\boldsymbol{c}$; green in $\boldsymbol{c}^{\prime \prime}$ ) in $\mathrm{KRH}$ for $15 \mathrm{~min}$ at $37^{\circ} \mathrm{C}$, fixed, and counterstained with an antibody against synapsin I ( $\boldsymbol{c}^{\prime}$; red in $\boldsymbol{c}^{\prime \prime}$ ). Synapsin I associates with Syt $t_{-}$-positive (i.e., recycling) SVs. Scale bar: (in $\left.\boldsymbol{a}\right) \boldsymbol{a}, 10 \mu \mathrm{m}$; b-c', $5 \mu \mathrm{m}$.

tected at synaptic varicosities, which at this developmental stage are only occasionally encountered in our cultures (Fig. $4 a$, arrowheads).

The finding that synapsins preferentially associate with a subpopulation of SVs prompted us to determine whether these vesicles displayed distinct properties. Neurons were allowed to internalize the $\mathrm{Syt}_{\mathrm{L}}$ antibody for $15 \mathrm{~min}$ in $\mathrm{KRH}$ and were subsequently counterstained for synapsin I. Remarkably, synapsin I showed an almost exclusive colocalization with constitutively recycling (i.e., Syt ${ }_{\mathrm{L}}$-positive) SVs (Fig. $4 c-c^{\prime \prime}$ ).

To investigate whether synapsin phosphorylation mediates the cAMP-dependent regulation of SV distribution and recycling in the growth cones, stage 3 neurons stimulated by either forskolin or $\mathrm{K}^{+}$-induced depolarization were triple labeled with antisynapsin antibodies that specifically recognize either phosphorylated site 1 (i.e., the PKA/CaMK I site), phosphorylated site 3 (i.e., one of the two CaMK II sites), or total synapsins. A parallel study of site 1 phosphorylation was performed by Western blot analysis of total lysates of cultured neurons followed by probing with site 1 phosphospecific anti-synapsin antibody (Fig. 5). A basal level of site 1 phosphorylation was present at rest and was strongly reduced by H89, a PKA inhibitor. In the growth cones, enhance-
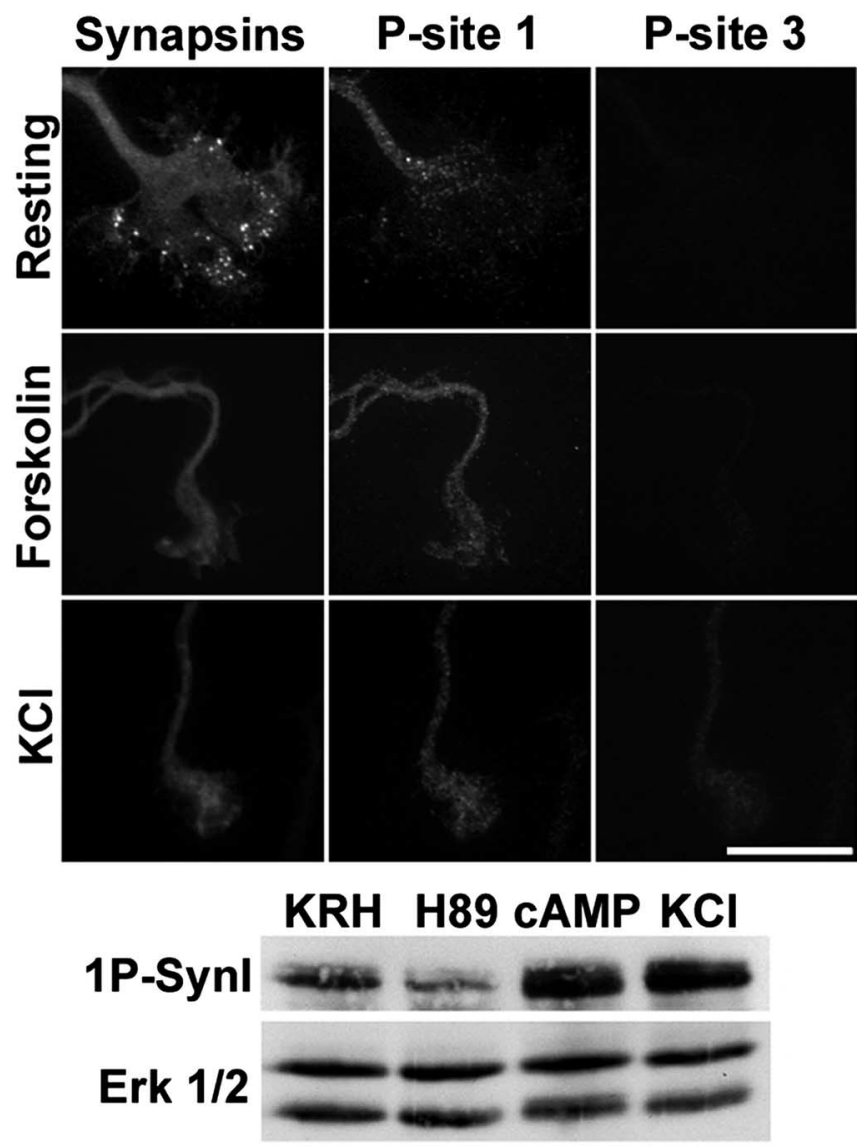

Figure 5. Synapsin is phosphorylated by PKA in growth cones. Top, Growth cones of rat hippocampal neurons incubated at $37^{\circ} \mathrm{C}$ in the presence or absence of either $50 \mu \mathrm{m}$ forskolin (for $10 \mathrm{~min}$ ) or $55 \mathrm{~mm} \mathrm{KCl}$ (for $1 \mathrm{~min}$ ), fixed, and processed for triple immunofluorescence with site 1 (P-site 1) and site 3 (P-site 3) phosphospecific anti-synapsin antibodies and with an antibody that recognizes total synapsins. At rest, a low level of basal site 1 phosphorylation is detectable. Phosphorylation at site 1 is enhanced by both forskolin and $\mathrm{KCl}$, whereas site 3 is phosphorylated weakly and exclusively during depolarization. Note that both treatments lead to the disappearance of the synapsin puncta visible in the control. Scale bar, $10 \mu \mathrm{m}$. Bottom, Cultured rat hippocampal neurons were lysed under control conditions (KRH) or after treatment

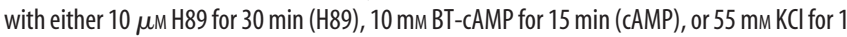
$\min (\mathrm{KCl})$. Equal amounts of protein were loaded into each lane. Parallel samples were probed with either a site 1 phosphospecific anti-synapsin antibody or an anti-ERK1/2 antibody. Basal site 1 phosphorylation is reduced by $\mathrm{H} 89$ treatment and enhanced by both BT-CAMP and depolarization.

ment of site 1 phosphorylation was induced by both forskolin and depolarization. BT-cAMP also promoted site 1 phosphorylation in total cell extracts. Consistent with previous reports, site 3 was virtually unphosphorylated under basal conditions and after treatment with forskolin and was only weakly activated after depolarization-induced $\mathrm{Ca}^{2+}$ influx in growth cones and developing axons (Jovanovic et al., 2001; Menegon et al., 2002). It is of interest to note that puncta of synapsin immunoreactivity were detected at rest, whereas after the application of stimuli that enhance site 1 phosphorylation a diffuse pattern of synapsin fluorescence was present, possibly because of dissociation of synapsin from SVs after phosphorylation (see below).

Phosphorylation at site 1 controls the state of association of synapsin I with SVs in the growth cone

To determine whether in the growth cones cAMP-dependent synapsin phosphoryation at site 1 is followed by dissociation from SVs, neurons incubated with forskolin were double stained 
for synaptotagmin I and synapsin I (Fig. 6, top). At rest, synapsin colocalized with SVs in the $\mathrm{C}$ domain. After 3 min of treatment with forskolin, synapsin puncta were no longer detected, although synaptotagmin I-positive SVs were still clearly visible in the $\mathrm{C}$ domain and virtually absent from the $\mathrm{P}$ domain. In contrast, when the stimulation was prolonged for $10 \mathrm{~min}$, mobilization of SVs into the $\mathrm{P}$ domain was also observed. This indicates that cAMPinduced synapsin dissociation from SVs precedes SV dispersion in the growth cone. At variance, synapsins remained associated with dispersed SVs after treatment with cytochalasin D (data not shown). Hippocampal neurons derived from mice lacking the synapsin I gene (Li et al., 1995) were coinfected with lentiviruses engineered to express SypI-EYFP and fusion proteins of ECFP with either wild-type synapsin Ia (ECFP-SynI) or a mutant lacking phosphorylation site 1 (ECFP-SynI S9A) under the control of the PGK promoter. To provide a semiquantitative evaluation of the colocalization between SypIEYFP and the synapsin chimeras, we measured the relative fluorescence intensities along arbitrary lines in growth cones and preterminal axons. Under resting conditions, the intensity profiles of SypIEYFP and of either synapsin chimera were highly superimposable, consistent with the idea that both wild-type and mutant synapsin I bind to SVs (Fig. 6, bottom). After forskolin treatment, ECFP-SynI displayed a uniform distribution of fluorescence intensity with no correlation with the peaks of SypI-EYFP fluorescence. In contrast, the intensity profiles of ECFPSynI S9A fluorescence still matched those of SypI-EYFP, indicating that the state of association of synapsin I with SVs in the growth cone is entirely dependent on site 1 phosphorylation.

\section{cAMP-modulated phosphorylation of} synapsin I at site 1 controls the distribution of SVs in the C domain of the growth cone

We next investigated whether the changes in SV distribution and the dissociation of synapsin I from SVs in response to elevation of intracellular cAMP are causally related events. Firstly, SV distribution was altered in most growth cones of hippocampal neurons from synapsin I knockout mice $(80 \% ; n=70)$. In the absence of synapsin I, synaptotagmin I-positive SVs did not concentrate in the C domain but appeared to be distributed throughout the growth cone, reaching the distal edge of the $\mathrm{P}$ domain. A similar distribution was observed in the growth cones of neurons prepared from synapsin I/II/III triple knock-out mice (average growth cone area occupied

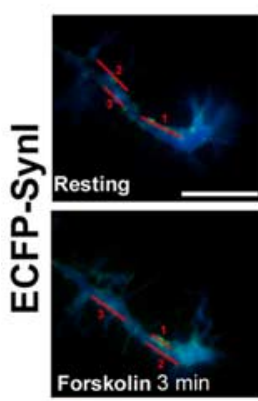

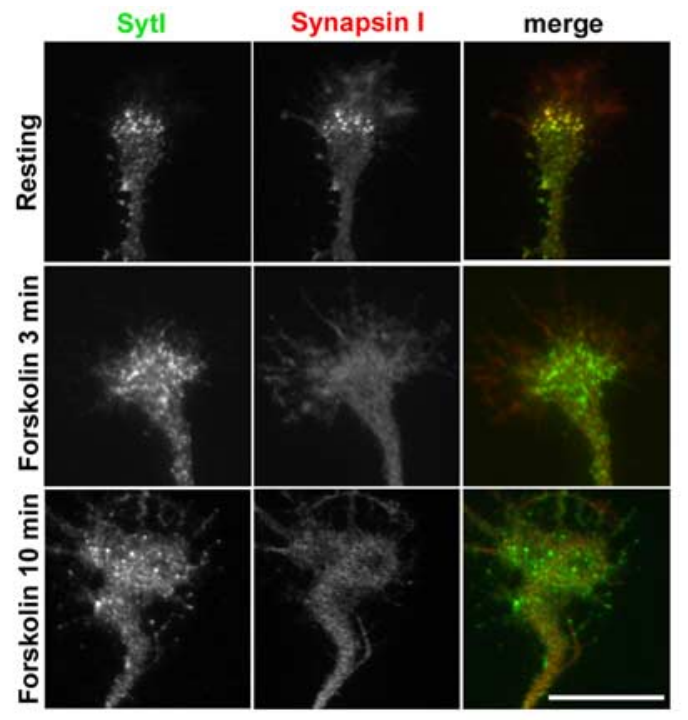
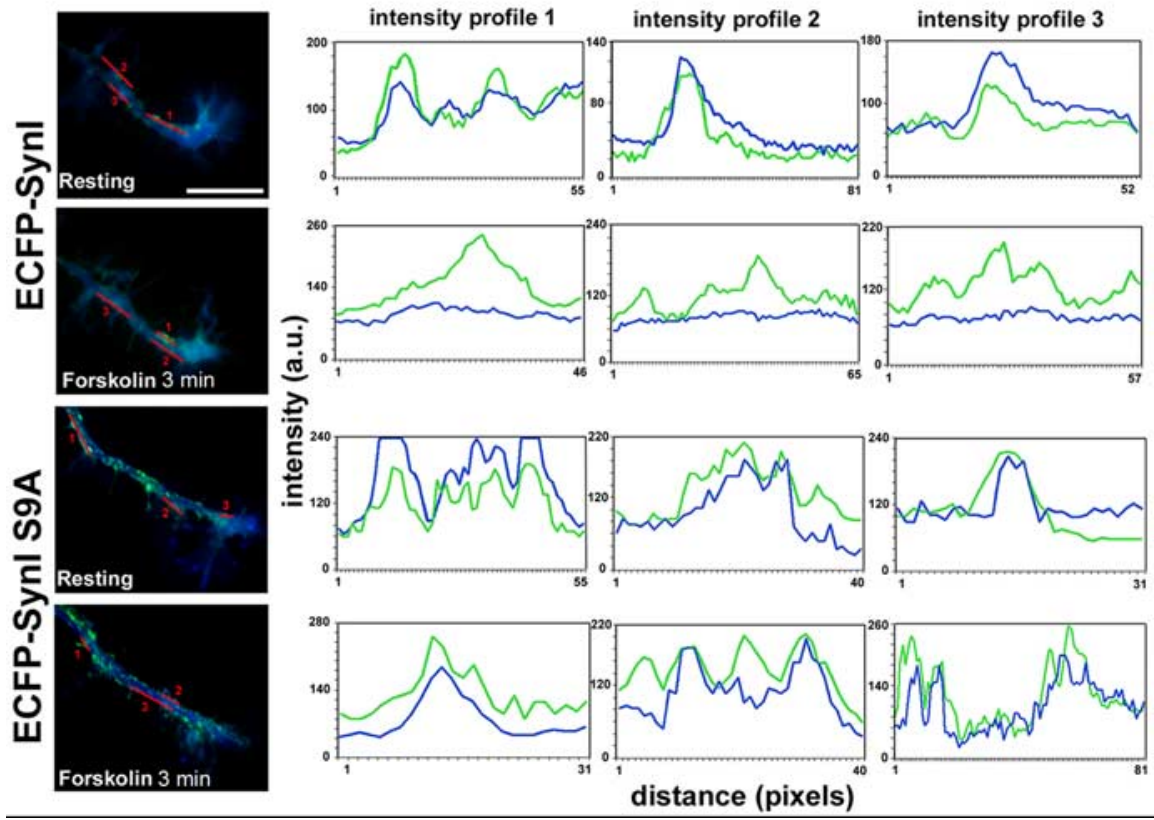

Figure 6. Phosphorylation at site 1 is required for the dissociation of synapsin I from SV s in the growth cone. Top, Growth cones of rat hippocampal neurons incubated for either 3 or $10 \mathrm{~min}$ at $37^{\circ} \mathrm{C}$ in the presence or absence of forskolin, fixed, and labeled with anti-synaptotagmin I (Sytl; green in the merged images) and anti-synapsin I (red in the merged images) antibodies. At rest, synapsin I colocalizes with a fraction of synaptotagmin I-positive SVs, but after forskolin treatment, it appears evenly distributed throughout the growth cone. Note that, 3 min after forskolin application, SVs are still clustered in the ( domain, whereas they become dispersed in the P domain when the stimulation is prolonged for $10 \mathrm{~min}$. Scale bar, $10 \mu \mathrm{m}$. Bottom, Growth cones and preterminal axons of hippocampal neurons from synapsin I knock-out mice double infected to express Sypl-EYFP (green) and either ECFP-Synl or ECFP-SynI S9A (blue), imaged both before and after incubation with $50 \mu \mathrm{m}$ forskolin for $3 \mathrm{~min}$. The fluorescence intensity plots of Sypl-EYFP (green traces) and of either ECFP-Synl or ECFP-SynI S9A (blue traces) measured along each of the numbered red lines are shown. Before treatment, the intensity peaks of both synapsin chimeras are mostly superimposed with those of Sypl-EYFP. After forskolin application, the ECFP-SynI S9A trace still peaks together with the Sypl-EYFP trace, whereas the traces of ECFP-Synl and Sypl-EYFP no longer overlap, and ECFP-Synl becomes uniformly distributed. Note that the position of the lines has been modified after forskolin incubation because of the changes occurred over time in SV localization. a.u., Arbitrary units. Scale bar, $10 \mu \mathrm{m}$.

by synaptotagmin I-positive SVs $\pm \mathrm{SD}, 78 \pm 13 \% ; n=35)$, whereas in neurons prepared from synapsin II single knock-out mice SVs were clustered in the core of the growth cone, thus resembling the situation observed in wild-type animals (data not shown).

Remarkably, expression of either ECFP-SynI or ECFP-SynI 
Synl KO
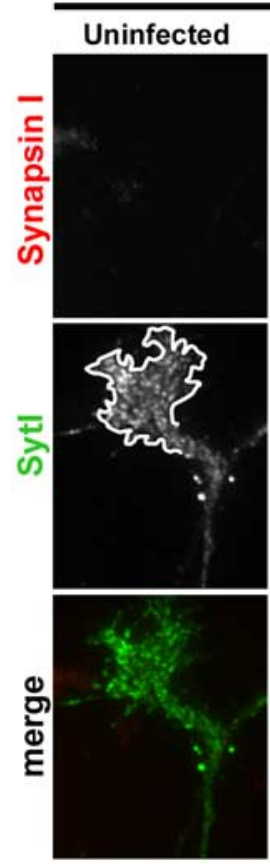
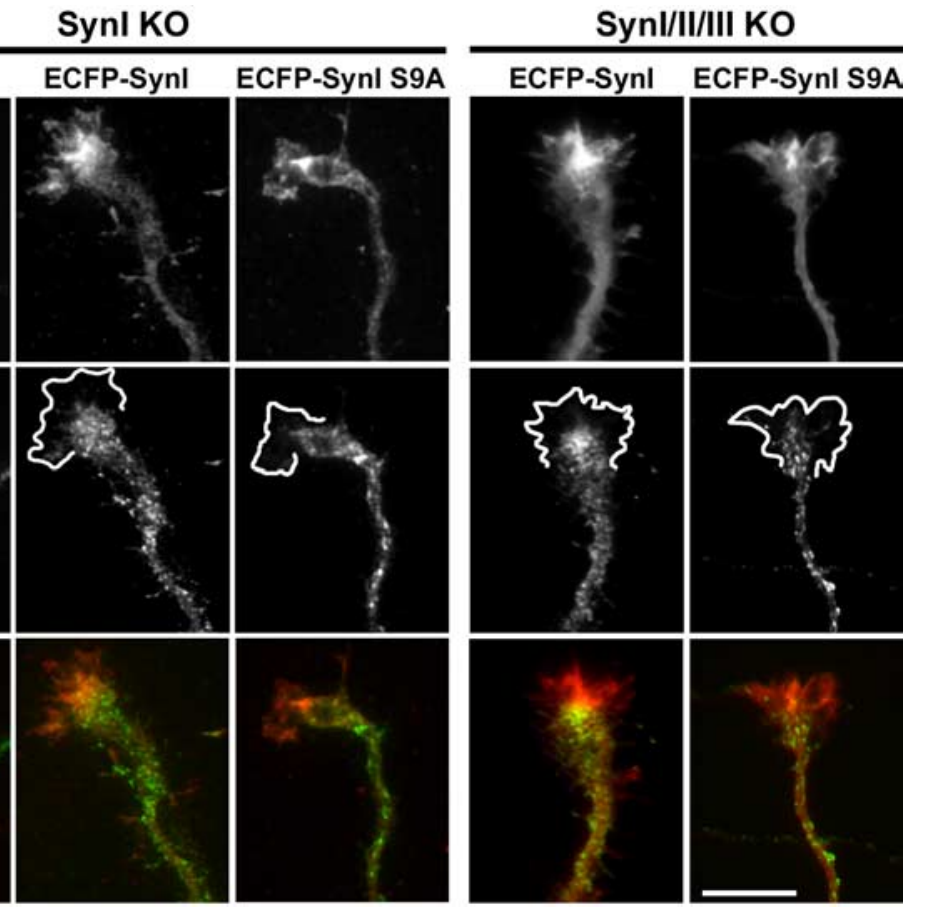

either uninfected growth cones or growth cones expressing either ECFP-SynI or ECFP-VAMP2 (Fig. 9). This indicates that
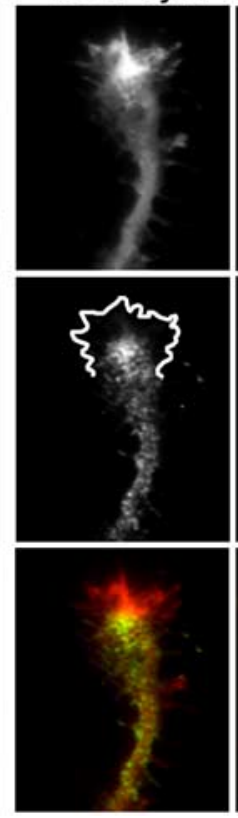

Figure 7. Synapsin I controls SV distribution in the C domain of the growth cone. Hippocampal neurons from either synapsin I knock-out (K0) mice (left) or synapsin I/I/III triple knock-out mice (right) infected with either ECFP-Synl or ECFP-SynI S9A or left uninfected, fixed, and stained with anti-synapsin (red in the merged images) and anti-synaptotagmin I (Sytl; green in the merged images) antibodies. In the infected cells, the synapsin antibody recognizes both synapsin chimeras, whereas no synapsin immunoreactivity is detected in the uninfected sample. Synaptotagmin I-positive SVs are distributed throughout the growth cone in uninfected neurons but confined to the C domain in both ECFP-Synl- and ECFP-Synl S9A-expressing growth cones. The white traces in the middle row outline the distal edges of the P domain, as determined based on DIC images. Scale bar, $10 \mu \mathrm{m}$.

S9A restored the localization of SVs in the C domain of the growth cone of both synapsin I knock-out neurons (ECFP-SynI, 93\%; ECFP-SynI S9A, 95\%; $n=45$ ) and synapsin I/II/III triple knock-out neurons (ECFP-SynI, 89\%, ECFP-SynI S9A, 94\%, $n=55$; average growth cone area occupied by synaptotagmin I-positive SVs \pm SD, ECFP-SynI, $41 \pm 14 \%$, ECFP-SynI S9A, $33 \pm 10 \%, n=30$ ) (Fig. 7). Thus, both wild-type synapsin I and its mutant lacking phosphorylation site 1 promote clustering of SVs in the C domain of the growth cone under basal conditions.

Interestingly, treatment with either forskolin or BT-cAMP led to dispersion of SVs in growth cones expressing wild-type synapsin I but was ineffective in those expressing the synapsin I S9A mutant (Fig. 8 and data not shown). Thus, phosphorylation of synapsin I at site 1 is required to allow dynamic changes in SV distribution in response to intracellular cAMP increases.

\section{Phosphorylation of synapsin I at site 1 controls the rate of basal SV recycling in the growth cone}

To test whether synapsin mediates the cAMP-dependent control of basal SV recycling, rat hippocampal neurons were infected with lentiviruses engineered to express either ECFP-SynI or ECFP-SynI S9A under the control of the CMV promoter, which allows to obtain high expression levels of the recombinant proteins. Uninfected neurons or neurons expressing the SV marker synaptobrevin 2/VAMP2 fused to ECFP were used as controls. Basal SV recycling in these neurons was studied by quantitative analysis of Syt $_{\mathrm{L}}$ antibody uptake during a 15 min incubation in KRH. Expression of ECFP-SynI did not affect SV recycling. In contrast, constitutive SV recycling in ECFP-SynI S9A expressing growth cones was strongly reduced when compared with recycling in
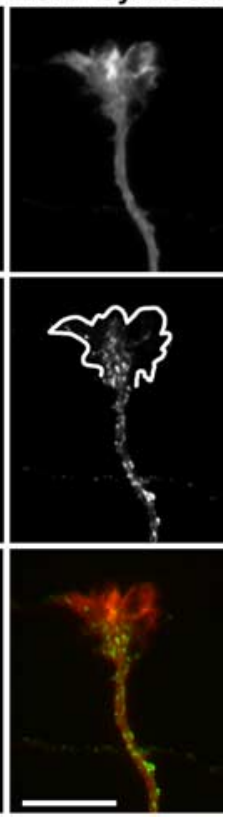

synapsin I S9A, which lacks phosphorylation site 1 , exerts a dominant-negative effect on the basal rate of SV recycling in the growth cones.

\section{Discussion}

In this study, we show that synapsin I plays a central role in regulating the organization and dynamics of SVs in growth cones and that this activity is modulated by cAMP-dependent phosphorylation of the protein.

The axonal growth cone is a site of intense membrane trafficking (Diefenbach et al., 1999). However, thus far, the possibility that the trafficking of the various organelles present in this compartment is differentially regulated had not been addressed. Using the lipophilic dye FM 4-64, we have visualized an intense process of membrane recycling characterized by rapid kinetics. However, SVs do not appear to be engaged in such fast recycling, and their exo-endocytosis occurs at a much slower rate, which can be visualized by uptake of the $S_{\mathrm{L}} \mathrm{L}_{\mathrm{L}}$ antibody. The relative stillness of SVs, which appear to be retained in the central core of the growth cone, is suggestive of the existence of a control device operating before the formation of synaptic contacts to limit SV recycling. Thus, the basal rate of SV exocytosis, which is likely to mediate the process of neurotransmitter release and hence neuronal communication already at these early stages, is dissociated from the general process of constitutive exocytosis of plasma membrane precursor vesicles and other ill-defined organelles, which leads to plasma membrane expansion and neurite outgrowth (Lockerbie et al., 1991; Leoni et al., 1999). In developing neurons, this inhibitory control is specific for growth cones, because SV precursors traveling down the axon have been reported to recycle at high rate (Matteoli et al., 1992). Localization of SVs in the $\mathrm{C}$ domain of the growth cone requires an intact F-actin meshwork. Cytochalasin D-induced disruption of the F-actin meshwork allows microtubule and cytoplasmic components to enter the P domain (Forscher and Smith, 1988; Contestabile et al., 2003). We observed that even relatively short exposures to cytochalasin D induce mobilization of both SVs and the endosomal marker syntaxin 13 from the $\mathrm{C}$ to the $\mathrm{P}$ domain (data not shown). This indicates that $\mathrm{F}$-actin represents an unspecific barrier that controls organelle distribution in the growth cone.

According to previous reports, cAMP enhances axonal anterograde transport, eventually inducing a $\mathrm{Ca}^{2+}$-independent redistribution of organelles into the $\mathrm{P}$ domain of the growth cone (Forscher et al., 1987; Hollenbeck, 1993). This effect was proposed to depend on the PKA-stimulated entry of microtubules and associated organelles into the $\mathrm{P}$ domain, possibly facilitated by cAMP-dependent loosing of the F-actin meshwork at the C-P domain interface (Forscher et al., 1987). To increase intracellular cAMP, we applied protocols milder than those used previously to produce a general enhancement of axonal anterograde transport 


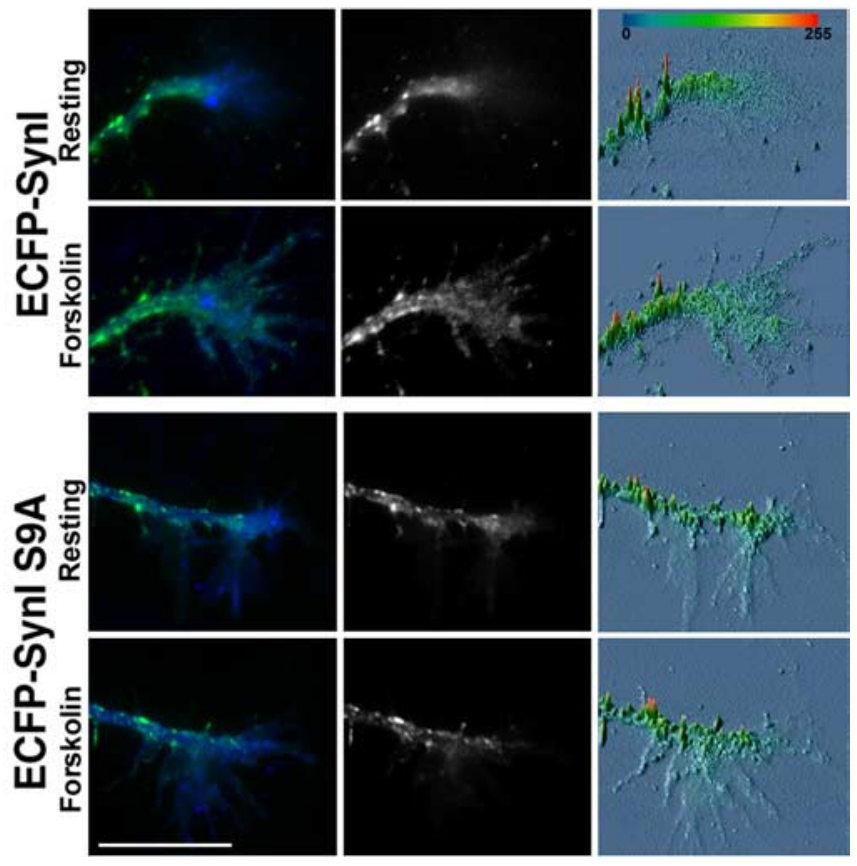

Figure 8. Site 1 phosphorylation of synapsin $\mathrm{I}$ is required for cAMP-modulated mobilization of SVs from the C domain of the growth cone. Growth cones of hippocampal neurons derived from synapsin I knock-out mice coexpressing Sypl-EYFP (green in the left column) and either ECFP-Synl or ECFP-SynI S9A (blue in the left column), imaged before and after incubation with $50 \mu \mathrm{m}$ forskolin for $5 \mathrm{~min}$ at RT. The middle column shows Sypl-EYFP fluorescence extracted from the merged images. In the right column, Sypl-EYFP image gray scales were transposed into a pseudocolor spectrum surface plot, with warmer hues corresponding to pixels of higher fluorescence intensity. Forskolin induces dispersion of Sypl-EYFP-bearing SVs in growth cones expressing ECFP-Synl but not in those expressing ECFP-Synl S9A. Scale bar, $10 \mu \mathrm{m}$.

(Forscher et al., 1987; Hollenbeck, 1993). Under these conditions, we observed selective changes in the distribution of SVs, which were accompanied by an enhancement in the rate of SV recycling. In contrast, the localization of syntaxin 13-positive endosomes in the distal axon was unaffected. No major modifications in F-actin organization and growth cone morphology were visible, arguing against a direct effect of cytoskeletal dynamics in the cAMP-stimulated changes in SV distribution and indicating that depolymerization of F-actin is sufficient but not necessary for redistribution of SVs to the distal domain of the growth cone.

We found that the lack of expression of synapsin I impairs the ability of the growth cone to retain SVs in the C domain under basal conditions. Reintroduction of synapsin I restores the correct localization of SVs. This effect does not require the formation of heterodimers between synapsin I and the other synapsin isoforms (Hosaka and Sudhof, 1999), because it can be reproduced by heterologous synapsin I expressed in synapsin I/II/III triple knock-out neurons.

These findings indicate that the SV clustering (Benfenati et al., 1993) and actin tethering (Valtorta et al., 1992) effects of synapsin I are required to maintain the compartmentalization of SVs within the growth cone. Moreover, the presence of a serine residue in position 9, corresponding to the unique PKA phosphorylation site, is necessary to cause dispersion of the vesicles after increases in intracellular cAMP.

Although we cannot rule out the possibility that other presynaptic proteins participate in the cAMP-mediated control of SV dynamics in the growth cone, phosphorylation of synapsin I by PKA appears to play a major role in this phenomenon. cAMP is known to promote $\mathrm{Ca}^{2+}$ entry in the presynaptic terminal at the
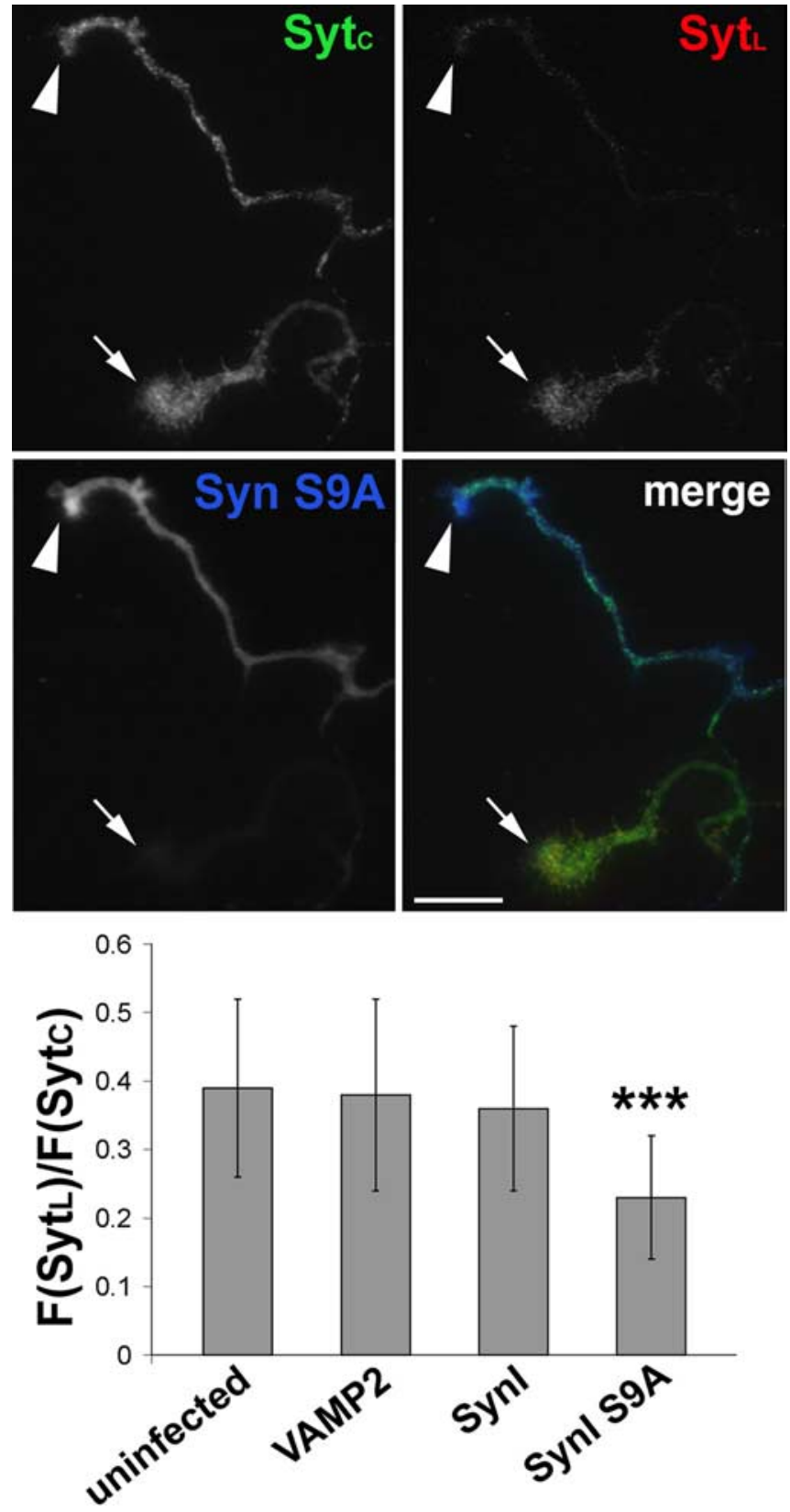

Figure 9. Site 1 phosphorylation of synapsin I controls the rate of $S V$ recycling in the growth cone. Top, Growth cones of rat hippocampal neurons infected with ECFP-SynI S9A (blue in the merged image) incubated with the Syt antibody (red in the merged image) in KRH for 15 min at $37^{\circ} \mathrm{C}$, fixed, and counterstained with an antibody against total synaptotagmin I (Syt ${ }_{c}$; green in the merged image). The arrowheads and the arrows point to ECFP-Synl S9A-infected and uninfected growth cones, respectively. Scale bar, $10 \mu \mathrm{m}$. Bottom, Quantitative analysis of basal Syt, internalization in growth cones expressing ECFP-VAMP2, ECFP-Synl, or ECFP-Synl S9A. The ratio between the fluorescence intensities of $S_{y} t_{L}$ and $S_{y} t_{C}$ in each growth cone is reported (mean $\pm S D ; n=50$ ). The internalization of Syt $t_{L}$ antibody is reduced in the ECFP-Synl S9Aexpressing growth cones $\left({ }^{* * *} p<0.001\right.$; Student's $t$ test; ECFP-SynI S9A vs uninfected growth cone).

neuromuscular junction (Funte and Haydon, 1993). However, it is unlikely that the observed effects are caused by cAMP-induced activation of $\mathrm{Ca}^{2+} /$ calmodulin-dependent kinases. Indeed, synapsin phosphorylation by CaMK II at site $2 / 3$ is undetectable in developing axons (Fig. 5) (Menegon et al., 2002), and studies with kinase inhibitors indicate that CaMK I has a limited role in the control of synapsin function mediated by site 1 phosphoryla- 
tion (A. Menegon, D. Bonanomi, and F. Valtorta, unpublished observations).

Our results suggest that the same molecular mechanisms that are at the basis of the role of synapsin in modulating neurotransmitter release from mature nerve terminals underlie also the role of the protein in SV dynamics in growth cones. Indeed, synapsin phosphorylation is regulated by synaptic activity (Nestler and Greengard, 1982; Sihra et al., 1989) and is associated with profound changes in its affinity for SVs and actin (Benfenati et al., 1989; Valtorta et al., 1992; Hosaka et al., 1999). Consistently, phosphorylated synapsin I dissociates from SVs and diffuses in the axon during high-frequency stimulation (Torri Tarelli et al., 1992; Chi et al., 2001). Thus, in mature nerve terminals, synapsin phosphorylation regulates the tethering of SVs to the actin cytoskeleton, leading to changes in the availability of SVs for exocytosis. Similarly, in growth cones, PKA-mediated phosphorylation of synapsin I regulates the spatial organization of SVs and their ability to undergo exo-endocytosis.

Synapsins appear to control the time course of synapse formation during development. Synapsin suppression delays neuronal differentiation, axonal extension, and synaptogenesis (Chin et al., 1995; Ferreira et al., 1995), whereas the precocious expression of synapsins leads to accelerated maturation of synapses formed in culture (Lu et al., 1992; Schaeffer et al., 1994; Valtorta et al., 1995; Fiumara et al., 2004). Exogenous synapsin I loaded into embryonic Xenopus spinal neurons induces profound structural rearrangements of the developing neuromuscular synapses, including the precocious compartmentalization of SVs in nerve terminals, accompanied by the early appearance of a mature form of quantal secretion (Lu et al., 1992; Valtorta et al., 1995).

We now show that both wild-type and mutated synapsin I are able to retain SVs in the C domain, but only the wild-type molecule can disperse SVs throughout the growth cone and promote their recycling. These findings suggest that the activity of synapsin I in promoting synapse maturation critically depends on its ability to respond to increases in intracellular cAMP and not simply on a structural effect in the spatial organization of organelles in the growth cone.

Because contact with the postsynaptic target retrogradely induces elevations in cAMP in the presynaptic compartment (Funte and Haydon, 1993), it is tempting to speculate that during the formation of neuronal circuits, synapsins translate the cAMP increase into the control of SV distribution and recycling, thus allowing SVs to participate in the structural and functional changes underlying the transformation of the growth cone into a mature secretory terminal.

The possibility that alterations in synapsin I functions lead to impairments in brain development and/or activity is a testable hypothesis. Indeed, synapsin I knock-out mice exhibit spontaneous seizures ( $\mathrm{Li}$ et al., 1995), and mutations in the synapsin I gene have been reported recently to cause epilepsy and/or mental retardation in humans (Garcia et al., 2004).

\section{References}

Ahmari SE, Buchanan J, Smith SJ (2000) Assembly of presynaptic active zones from cytoplasmic transport packets. Nat Neurosci 3:445-451.

Banker GA, Cowan WM (1977) Rat hippocampal neurons in dispersed cell culture. Brain Res 126:397-442.

Benfenati F, Bahler M, Jahn R, Greengard P (1989) Interactions of synapsin I with small synaptic vesicles: distinct sites in synapsin I bind to vesicle phospholipids and vesicle proteins. J Cell Biol 108:1863-1872.

Benfenati F, Valtorta F, Rossi MC, Onofri F, Sihra T, Greengard P (1993) Interactions of synapsin I with phospholipids: possible role in synaptic vesicle clustering and in the maintenance of bilayer structures. J Cell Biol 123:1845-1855.

Chi P, Greengard P, Ryan TA (2001) Synapsin dispersion and reclustering during synaptic activity. Nat Neurosci 4:1187-1193.

Chin LS, Li L, Ferreira A, Kosik KS, Greengard P (1995) Impairment of axonal development and of synaptogenesis in hippocampal neurons of synapsin I-deficient mice. Proc Natl Acad Sci USA 92:9230-9234.

Contestabile A, Bonanomi D, Burgaya F, Girault JA, Valtorta F (2003) Localization of focal adhesion kinase isoforms in cells of the central nervous system. Int J Dev Neurosci 21:83-93.

Diefenbach TJ, Guthrie PB, Stier H, Billups B, Kater SB (1999) Membrane recycling in the neuronal growth cone revealed by FM1-43 labeling. J Neurosci 19:9436-9444.

Ellis L, Katz F, Pfenninger KH (1985) Nerve growth cones isolated from fetal rat brain. II. Cyclic adenosine $3^{\prime}, 5^{\prime}$-monophosphate (cAMP)-binding proteins and cAMP-dependent protein phosphorylation. J Neurosci 5:1393-1401.

Feng J, Chi P, Blanpied TA, Xu Y, Magarinos AM, Ferreira A, Takahashi RH, Kao HT, McEwen BS, Ryan TA, Augustine GJ, Greengard P (2002) Regulation of neurotransmitter release by synapsin III. J Neurosci 22:4372-4380.

Ferreira A, Han HQ, Greengard P, Kosik KS (1995) Suppression of synapsin II inhibits the formation and maintenance of synapses in hippocampal culture. Proc Natl Acad Sci USA 92:9225-9229.

Fiumara F, Giovedi S, Menegon A, Milanese C, Merlo D, Montarolo PG, Valtorta F, Benfenati F, Ghirardi M (2004) Phosphorylation by cAMPdependent protein kinase is essential for synapsin-induced enhancement of neurotransmitter release in invertebrate neurons. J Cell Sci 117:5145-5154.

Forscher P, Smith SJ (1988) Actions of cytochalasins on the organization of actin filaments and microtubules in a neuronal growth cone. J Cell Biol 107:1505-1516

Forscher P, Kaczmarek LK, Buchanan JA, Smith SJ (1987) Cyclic AMP induces changes in distribution and transport of organelles within growth cones of Aplysia bag cell neurons. J Neurosci 7:3600-3611.

Funte LR, Haydon PG (1993) Synaptic target contact enhances presynaptic calcium influx by activating cAMP-dependent protein kinase during synaptogenesis. Neuron 10:1069-1078.

Garcia CC, Blair HJ, Seager M, Coulthard A, Tennant S, Buddles M, Curtis A, Goodship JA (2004) Identification of a mutation in synapsin I, a synaptic vesicle protein, in a family with epilepsy. J Med Genet 41:183-186.

Hilfiker S, Schweizer FE, Kao HT, Czernik AJ, Greengard P, Augustine GJ (1998) Two sites of action for synapsin domain $\mathrm{E}$ in regulating neurotransmitter release. Nat Neurosci 1:29-35.

Hilfiker S, Pieribone VA, Czernik AJ, Kao HT, Augustine GJ, Greengard P (1999) Synapsins as regulators of neurotransmitter release. Philos Trans R Soc Lond B Biol Sci 354:269-279.

Hollenbeck PJ (1993) Products of endocytosis and autophagy are retrieved from axons by regulated retrograde organelle transport. J Cell Biol 121:305-315.

Hosaka M, Sudhof TC (1999) Homo- and heterodimerization of synapsins. J Biol Chem 274:16747-16753.

Hosaka M, Hammer RE, Sudhof TC (1999) A phospho-switch controls the dynamic association of synapsins with synaptic vesicles. Neuron 24:377-387.

Huttner WB, Greengard P (1979) Multiple phosphorylation sites in protein I and their differential regulation by cyclic AMP and calcium. Proc Natl Acad Sci USA 76:5402-5406.

Jovanovic JN, Benfenati F, Siow YL, Sihra TS, Sanghera JS, Pelech SL, Greengard P, Czernik AJ (1996) Neurotrophins stimulate phosphorylation of synapsin I by MAP kinase and regulate synapsin I-actin interactions. Proc Natl Acad Sci USA 93:3679-3683.

Jovanovic JN, Sihra TS, Nairn AC, Hemmings Jr HC, Greengard P, Czernik AJ (2001) Opposing changes in phosphorylation of specific sites in synapsin I during $\mathrm{Ca}^{2+}$-dependent glutamate release in isolated nerve terminals. J Neurosci 21:7944-7953.

Kao HT, Porton B, Hilfiker S, Stefani G, Pieribone VA, DeSalle R, Greengard P (1999) Molecular evolution of the synapsin gene family. J Exp Zool 285:360-377.

Koh TW, Bellen HJ (2003) Synaptotagmin I, a Ca2 + sensor for neurotransmitter release. Trends Neurosci 26:413-422.

Leoni C, Menegon A, Benfenati F, Toniolo D, Pennuto M, Valtorta F (1999) 
Neurite extension occurs in the absence of regulated exocytosis in PC12 subclones. Mol Biol Cell 10:2919-2931.

Li L, Chin LS, Shupliakov O, Brodin L, Sihra TS, Hvalby O, Jensen V, Zheng D, McNamara JO, Greengard P (1995) Impairment of synaptic vesicle clustering and of synaptic transmission, and increased seizure propensity, in synapsin I-deficient mice. Proc Natl Acad Sci USA 92:9235-9239.

Lockerbie RO, Miller VE, Pfenninger KH (1991) Regulated plasmalemmal expansion in nerve growth cones. J Cell Biol 112:1215-1227.

Lotti F, Menguzzato E, Rossi C, Naldini L, Ailles L, Mavilio F, Ferrari G (2002) Transcriptional targeting of lentiviral vectors by long terminal repeat enhancer replacement. J Virol 76:3996-4007.

Lu B, Greengard P, Poo MM (1992) Exogenous synapsin I promotes functional maturation of developing neuromuscular synapses. Neuron 8:521-529.

Matteoli M, Takei K, Perin MS, Sudhof TC, De Camilli P (1992) Exoendocytotic recycling of synaptic vesicles in developing processes of cultured hippocampal neurons. J Cell Biol 117:849-861.

Menegon A, Dunlap DD, Castano F, Benfenati F, Czernik AJ, Greengard P, Valtorta F (2000) Use of phosphosynapsin I-specific antibodies for image analysis of signal transduction in single nerve terminals. J Cell Sci 113:3573-3582.

Menegon A, Verderio C, Leoni C, Benfenati F, Czernik AJ, Greengard P, Matteoli M, Valtorta F (2002) Spatial and temporal regulation of $\mathrm{Ca}^{2+}$ / calmodulin-dependent protein kinase II activity in developing neurons. J Neurosci 22:7016-7026.

Nestler EJ, Greengard P (1982) Nerve impulses increase the phosphorylation state of protein I in rabbit superior cervical ganglion. Nature 296:452-454.

Pennuto M, Dunlap D, Contestabile A, Benfenati F, Valtorta F (2002) Fluorescence resonance energy transfer detection of synaptophysin I and vesicle-associated membrane protein 2 interactions during exocytosis from single live synapses. Mol Biol Cell 13:2706-2717.

Pennuto M, Bonanomi D, Benfenati F, Valtorta F (2003) Synaptophysin I controls the targeting of VAMP2/synaptobrevin II to synaptic vesicles. Mol Biol Cell 14:4909-4919.

Prekeris R, Foletti DL, Scheller RH (1999) Dynamics of tubulovesicular recycling endosomes in hippocampal neurons. J Neurosci 19:10324-10337.

Sabo SL, McAllister AK (2003) Mobility and cycling of synaptic protein- containing vesicles in axonal growth cone filopodia. Nat Neurosci 6:1264-1269.

Schaeffer E, Alder J, Greengard P, Poo MM (1994) Synapsin IIa accelerates functional development of neuromuscular synapses. Proc Natl Acad Sci USA 91:3882-3886.

Seamon KB, Padgett W, Daly JW (1981) Forskolin: unique diterpene activator of adenylate cyclase in membranes and in intact cells. Proc Natl Acad Sci USA 78:3363-3367.

Sihra TS, Wang JK, Gorelick FS, Greengard P (1989) Translocation of synapsin I in response to depolarization of isolated nerve terminals. Proc Natl Acad Sci USA 86:8108-8112.

Sudhof TC (2004) The synaptic vesicle cycle. Annu Rev Neurosci 27:509-547.

Torri Tarelli F, Bossi M, Fesce R, Greengard P, Valtorta F (1992) Synapsin I partially dissociates from synaptic vesicles during exocytosis induced by electrical stimulation. Neuron 9:1143-1153.

Trudeau LE, Emery DG, Haydon PG (1996) Direct modulation of the secretory machinery underlies PKA-dependent synaptic facilitation in hippocampal neurons. Neuron 17:789-797.

Valtorta F, Jahn R, Fesce R, Greengard P, Ceccarelli B (1988) Synaptophysin (p38) at the frog neuromuscular junction: its incorporation into the axolemma and recycling after intense quantal secretion. J Cell Biol 107:2717-2727.

Valtorta F, Greengard P, Fesce R, Chieregatti E, Benfenati F (1992) Effects of the neuronal phosphoprotein synapsin I on actin polymerization. I. Evidence for a phosphorylation-dependent nucleating effect. J Biol Chem 267:11281-11288.

Valtorta F, Iezzi N, Benfenati F, Lu B, Poo MM, Greengard P (1995) Accelerated structural maturation induced by synapsin I at developing neuromuscular synapses of Xenopus laevis. Eur J Neurosci 7:261-270.

Yao WD, Rusch J, Poo M, Wu CF (2000) Spontaneous acetylcholine secretion from developing growth cones of Drosophila central neurons in culture: effects of cAMP-pathway mutations. J Neurosci 20:2626-2637.

Young SH, Poo MM (1983) Spontaneous release of transmitter from growth cones of embryonic neurones. Nature 305:634-637.

Zakharenko S, Chang S, O’Donoghue M, Popov SV (1999) Neurotransmitter secretion along growing nerve processes: comparison with synaptic vesicle exocytosis. J Cell Biol 144:507-518. 